\title{
Museum Landscape Design based on the Combination of Art and Technology
}

\author{
Chunchen $\mathrm{Lu}^{1}$, xinyue Zhang ${ }^{2}$ \\ ${ }^{1,2}$ Lijiang College of Guangxi Normal University, Guilin, 541006, China
}

\begin{abstract}
With the view of solving the problems of development and innovation in museum landscape design, this paper, starting from the function of landscape, puts forward a strategy of combining art with science and technology. It takes static landscape and dynamic landscape as the starting point of design, and uses the design method of functional zoning optimization. It treats the fire museum as the theme, and makes landscape art modeling by using foam, cement, brick, steel wire and other materials. It combines intelligent fire control system and virtual technology to carry out fire escape experiment in art environment. The experimental result goes to show that artistic means are conducive to enhancing immersive experience, and scientific and technological means are conducive to improving the depth and dimension of experience. The museum landscape design based on the combination of art and science can help the value promotion of exhibition knowledge, landscape knowledge, exhibition hall education, science, art and interest. The combination of art and science and technology, which is the new trend of landscape design, provides a better comprehensive solution for the innovation in museum landscape design.
\end{abstract}

\section{Introduction}

The museum is a physical place to collect, collect, display and research the natural and human cultural heritage, and a cultural and educational institution and building that provides knowledge, education and appreciation of art for the public. Museums have become the main representatives of modern civilization and play a significant role in social life. For most visitors, visiting the museum itself, of course, is a spiritual baptism. In the exhibition design concerning museum space, the indoor and outdoor landscape design is very important. It plays an irreplaceable role in creating exhibition atmosphere, transmitting exhibition information and advocating cultural spirit. Thereby, it is widely concerned by the society. Suzhou Museum, beyond doubt, gives people static aesthetic enjoyment of traditional landscape. In the aspect of modern flavor, a problem with the integration of modern scientific and technological innovation and museum landscape design has been constantly explored by designers. And based on the future development trend, the author expresses the view of adopting the combination of modern technology and art in the aspect of museum landscape design.

\section{Basic understanding of museum landscape}

\subsection{The basic concept of museum landscape}

Landscape refers to landscape and scenery. From the perspective of design, "landscape", which is designed through human brain creativity, is a visual attraction for people to watch and enjoy. Because it is caused by human factors, it is different from the natural landscape. Landscape design is a kind of transformation behavior that people have consciousness to the specific environment by comprehensively considering various elements such as architecture, sculpture, garden, etc, and it is a kind of scenery that makes it have the value of form, form, culture and aesthetics. The garden landscape of museum refers to all spaces except the main building in the museum base, which adopts the method of landscape design to create a comfortable, relaxing, resting and pleasant landscape environment, in an effort to make nervous and tired visitors can relax and relax in the landscape.

\subsection{Functions of museum landscape}

Museum landscape has the functions of beautification, recreation and ecology.

2.2.1 Beautification function. The beautification function regarding museum landscape occupies an important position. When designing landscape, designers consider how to improve the aesthetic value of landscape in a natural way. The aesthetic system composed of human vision, emotional needs, natural and human factors of environment, dynamic and static factors of environment is an important basis for landscape design

*Corresponding author's e-mail: 3537348498@qq.com 
and research. The improvement of the landscape environment can stimulate people's vision by beautiful things, produce good emotions, facilitate the positive role of aesthetic, thus upgrading the aesthetic value. The original intention concerning landscape design is to give people aesthetic enjoyment of garden environment to the greatest degree, namely, landscape effect. Landscape effect refers to the relationship between the environment of aesthetic object and the mutual induction and transformation of aesthetic subject. The magnitude of the impact depends on two aspects. One is the role of human beings in the environment; the other is the role of the environment in human beings. The mutual induction and transformation between aesthetic environment and human beings give people the enjoyment of beauty by the expression in artistic conception ${ }^{[1]}$.

2.2.2 Leisure and relaxation function. As the audience is tired of seeing the exhibits in the museum, the physiological instinct will make the human vision naturally seek a natural, comfortable and elegant scenery to regulate the tired visual nerve. Therefore, the space of the museum should be reasonably designed, practical and functional, and meet the physiological needs of the audience. The space combination should be skillfully used, and the display should be integrated with the natural landscape and human landscape. When going out of the showroom, what the viewer sees, apart from the landscape, flowers and trees, are the bird's voice and floral fragrance, or the pavilion, or minimalist dry landscape. This can adjust the visual nerve of the audience, but at the same time it can relieve the visual fatigue, making people feel particularly friendly, thus achieving a kind of spiritual communication of blending feelings and scenes, and realizing relaxation and relaxation.

2.2.3 Ecological function. The landscape studied in this paper mainly refers to the landscape formed by human factors, but the success of the landscape design should also refer to its harmonious proportion with natural factors, because this proportion has become an important symbol of landscape design evaluation. It mainly represents the occupancy rate of green natural vision and the frequency of the alternation of man-made landscape and natural landscape. Therefore, the ecological function of landscape construction transforms the regional environment through protecting the regional ecological plant community and water system. It is reflected in the following aspects: first, improving the climate. A certain area of plants can regulate the ambient temperature and comfort in a certain area, improve the vertical ventilation of the museum, evacuate and weaken the heat island effect of the museum; secondly, purify the air in the museum to decrease noise pollution and improve the health environment. Greening and water resources are used as the main means to improve the microclimate in the museum, and the enhancement of environmental comfort is a reasonable embodiment of ecological functions.

\section{Museum landscape design}

\subsection{Integration of traditional landscape and modern design}

Chinese traditional landscape architects abide by the natural rules. Throughout the history of the Han Dynasty, the natural landscape garden of Taiyuan in the Qin and Han dynasties developed into the man-made garden of the prosperous city, all of which are based on the poetic and picturesque feelings of landscape and elegant garden. In terms of construction concept, there were aesthetic guiding ideology of "unity of nature and man" and "intelligent people enjoy water, benevolent people enjoy mountains". Nowadays, there are "more is less, less is more" new Chinese landscape techniques, such as "blank", "Silence", "void", "Zen" and so on, all of which highlight the creative idea of "landscape in painting, painting in landscape". Inheriting the traditional aesthetic construction idea of poetic and picturesque, the landscape design of museum environment emphasizes the design idea of "middle" and "new" of landscape and the harmonious modeling principle of "not high, not big and not outstanding". Suzhou Museum, as a landmark cultural building in the ancient city of Suzhou, shows the integration of traditional Chinese garden and modern design. It carries the profound historical and cultural heritage of Suzhou, and deduces the inheritance and innovation of art masterpieces. As shown in Figure 1, it is the north view of the main courtyard of Suzhou Museum. The rockeries placed with rubble are arranged in rows. The design inspiration is found from the ancient landscape paintings. The beautiful artistic conception of Mi Fu's ink and water paintings is created with the white wall as the background.

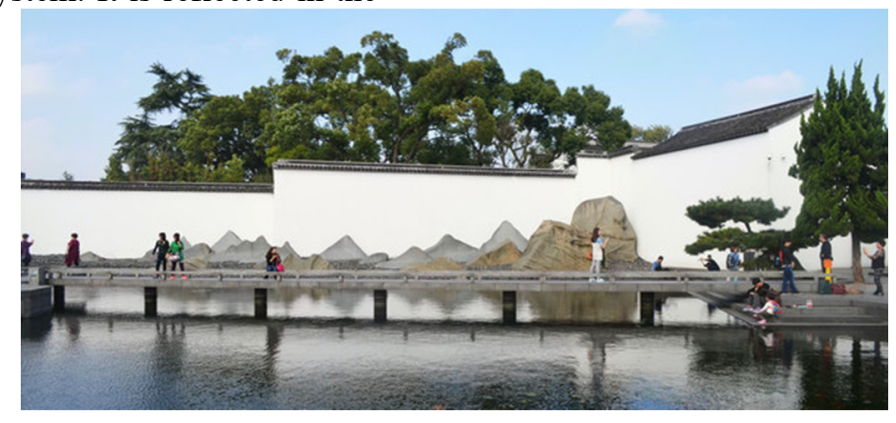

Figure 1. North view of the main courtyard of Suzhou Museum 


\subsection{Main problems in museum landscape design}

China's landscape design originated from the slave society and had gardening activities. Please refer to literature ${ }^{[2]}$, and the earliest museum design started in the thirty first year of Guangxu-1905 $5^{[3]}$. Zhang Jian established the first museum in China in Tongzhou, namely, Nantong Museum. The combination of landscape design and museum design only appears in modern society, people are familiar with music fountain landscape, city lighting night landscape, etc. As a result of the late start of landscape science and technology application research, there are still many problems that need to be solved in some aspects of design.

3.2.1. Limited form, blind plagiarism. Due to the influence of traditional landscape ideas in the 4000 year long history of landscape culture, many forms of landscape design are limited by traditional methods, lacking vitality and new breath. In modern times, the trend of learning from the West has sprung up in China. In terms of daily necessities and urban buildings, the situation of imitating the West has appeared. The same is true of landscape architecture. European style is everywhere. It should be noted that foreign excellent landscape design is worth learning, but blindly following the trend and copying the western style will backfire. For instance, in some second and third tier cities, European style buildings stand abruptly somewhere, which are not compatible with the surrounding buildings and destroy the original local architectural style. Thus, the reference must be based on the similarity of culture, geography, climate and other factors, and the appropriate imitation can improve the viewing effect in an effective way and have a good impact on the overall layout ${ }^{[4]}$.

\subsubsection{Ignore the science and technology of} museum landscape. The designer's thinking is intoxicated by the classical classic techniques and ignores the development and application of real science and technology due to the influence of traditional landscape design concept. They didn't fully understand the design function in museum landscape, blindly pursued classicality and inheritance, did not start from the perspective of development, and ignored the innovation and technology of modern garden landscape. Especially in the landscape design of the museum with modern theme as exhibition content, the innovation and technology of its design methods are very important.

\section{The strategy of the combination of art and technology in the design of museum landscape}

The museum landscape is mainly to meet the needs of the audience to contact and be close to the nature, relieve the pressure by participating in the contact of the natural environment, and improve the function regarding physical and mental state. As far as the important function of the museum landscape is concerned, its artistry exceeds its practicability. But in the aspect of strengthening the artistry of the landscape, it is a new trend for the development of the museum landscape to involve the scientific and technological means in the creation of the landscape.

In the integration of "technology" and "landscape", we should strictly follow the guidance of landscape planning, respect the natural environment, and combine intelligent technology to make the landscape fill the needs of the audience, and enjoy the fun brought by technology from different dimensions. On the basis of landscape aesthetics, it is primarily use the following two scientific and technological means to improve the experience interaction, multi-dimensional enhance the beauty of the landscape, in an effort to expand the influence of the museum.

\subsection{Landscape static shaping and technological breakthrough}

Traditionally, the appreciation of garden landscape is to get different views in the garden, through the tour route organized by spatial sequence, we can get the aesthetic enjoyment of "changing scenery while walking" by watching the scenery along the road. Suzhou Museum has "Mouth" and "Yi" shaped tour routes: the building is uniformly arranged along the surrounding area, facing the larger water surface in the center; before entering the main space, the view is gathered to get a sense of openness. ${ }^{[5]}$

The beauty of Suzhou Museum belongs to the static beauty of immersion, although people are traveling in the garden, the feeling of the beauty of the scenery they get is the same. They just appreciate it from a different angle. For the static beauty of landscape, it is usually to create the background with light and color. In the aspect of hearing, classical music is used to create an immersive experience environment, with silent water, quiet water curtain, misty smoke, and the piano likes a tide.... technology enhances the appeal of beauty.

The scientific and technological means of using light and color, smoke, intelligent music to create the garden mood and enhance the landscape atmosphere are more and more popular in many domestic and foreign tourism landscape applications. As shown in Figure 2, Las Vegas night scene, building the prosperity and charm of Las Vegas night scene with light and color. Figure 3 is a night view of two rivers and four lakes in Guilin, showing the quiet beauty of Lijiang River through light and color, such as Zahiram Duoduo's poem: if you see me or don't see me, I will be there, not sad or not happy; if you miss me or don't miss me, love will be there, not come or not go. Or, let you care about me, with silent, love, quiet and joy. 


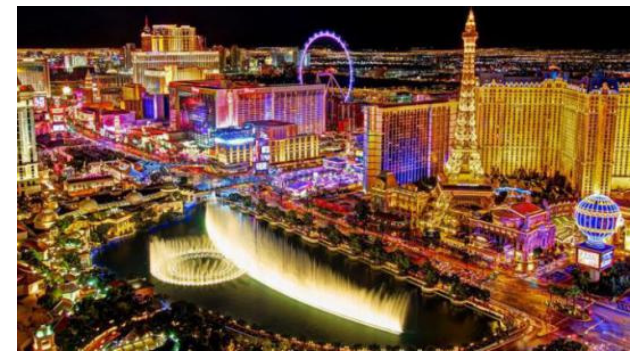

Figure 2. Las Vegas night scene ( source https://image.baidu.com)

\subsection{Landscape dynamic construction and technological breakthrough}

Landscape dynamic refers to the different aesthetic feelings produced by different changes of the same landscape point. The quiet lake scenery suddenly sounds music, and the fountains in the lake flow correspondingly. With the ups and downs of the music, there are changes in the form. The aesthetic pleasure generated by the appreciation process is caused by the dynamic changes of the landscape. There are many ways to introduce science and technology into the landscape of the museum in terms of dynamic changes, among which intelligent light color and intelligent music control light color and music through the distance between people and the landscape: when people just enter the landscape point, there will be a bright white warm light, and the music will also ring out. When people leave, the time line becomes dark, and the music stops.... Some museum landscapes are in dynamic simulation reality, such as volcanic eruption, cold wind, sleet, rainbow, birds, snakes and other dynamic animals, dynamic character scenes, and so on. There are many technologies in this kind of dynamic technology design, including human body temperature control and pressing delay. However, no matter what kind of science and technology, the shape and shape should be coordinated with the landscape environment.

\section{Case analysis of fire museum landscape}

\subsection{Site overview}

The Fire Museum of this case is located in Fuzhou City. The exhibition hall is divided into three parts: the first floor is about 400 square meters, the lower ground floor

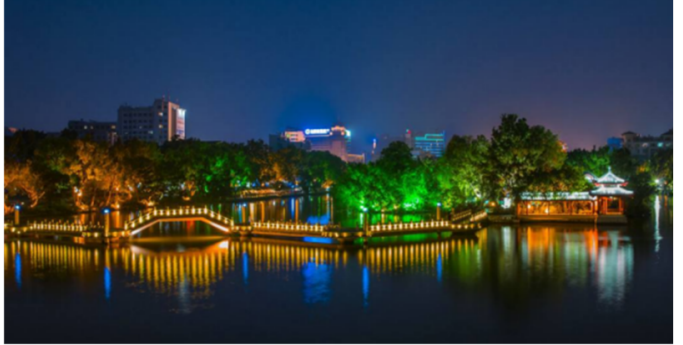

Figure 3. night view of Gui lake in Guilin ( source https://image.baidu.com)

is about 1200 square meters, and the third floor is about 300 square meters. There is a long and narrow stairway from the first floor to the lower floor. From the first floor to the third floor, you need to walk two floors with a relatively large loudness.

\subsection{Design concept}

The contents concerning the exhibition are the objects of the fire fighting system and the fire fighting publicity, fire museum landscape design should pay special attention to the stairway outside the exhibition hall for landscape design, and focus on the interior space landscape design. The design should display the concept of fire protection theme, and the exhibition mode should be blended with digital and intelligent technology.

\subsection{Space construction}

The functional layout of the exhibition area is: the first floor is the entrance of the exhibition hall, with intelligent security check. As shown in Figure 4, the lower ground floor is the fire station area, displaying the ancient and modern fire fighting. As shown in Figure 5, the third floor is equipped with modern fire fighting and interactive experience projects. As shown in Figure 6, three interior spaces are exhibition areas. The narrow passage from the first floor to the lower ground floor and the long stairs from the first floor to the third floor are the outdoor landscape areas. In the design psychology of the whole exhibition hall, the spatial sequence technique of "raising - restraining - raising" is adopted, which makes the visitors' mood produce the effect of "moving the scenery while walking" along with the visiting process. 


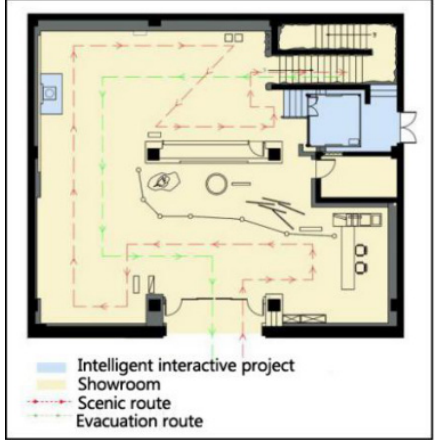

Figure 4. entrance of exhibition hall

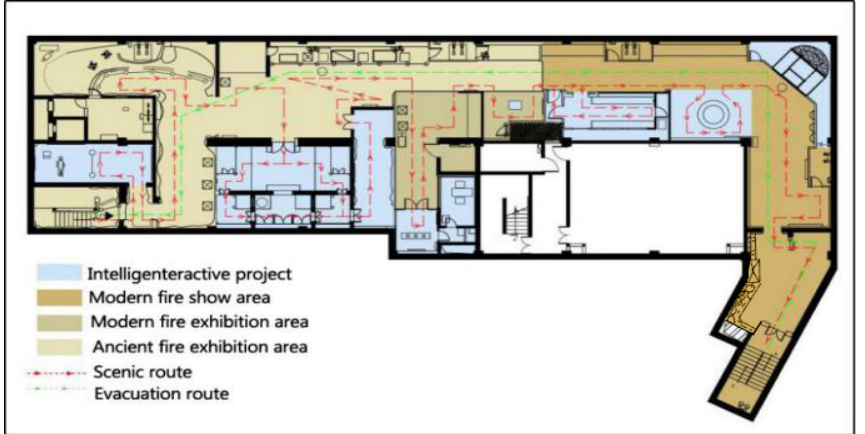

Figure 5. fire station area

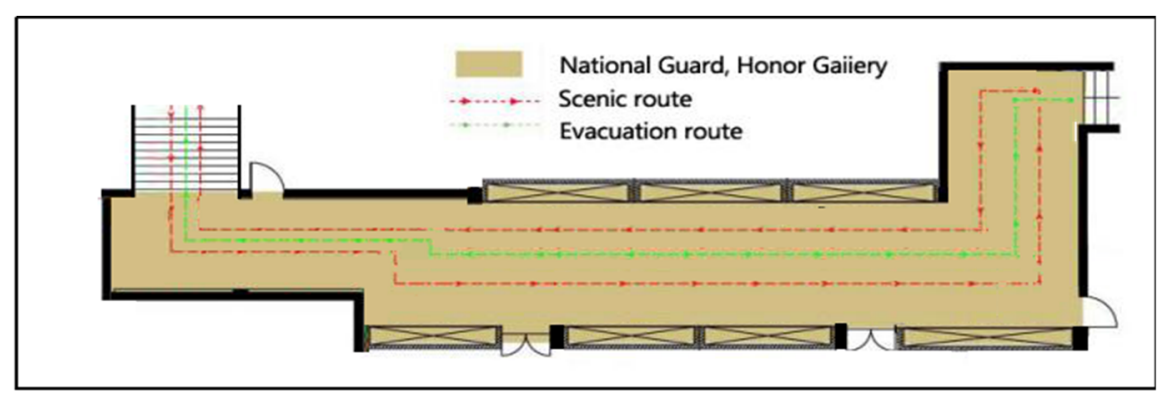

Figure 6. fire experience area

\subsection{Integration of art and technology}

5.4.1. Half pavilion sound image. At the entrance of the fire fighting exhibition area on the first floor of the basement, because of the wide space, the width is 5.6 meters at most, an ancient outdoor architectural landscape is designed. In terms of artistic expression, a pavilion with six and a half corners connected to the wall is built. In the pavilion, there is a robot imitating the ancient people's life scene to avoid heat. Around the pavilion, there are poultry, livestock, fenced plants and other space models. Thus the cheering and birdsong of farm chickens and dogs based on Multimedia Technology can be heard, and a scene of rural life in ancient times has jumped into front of us.

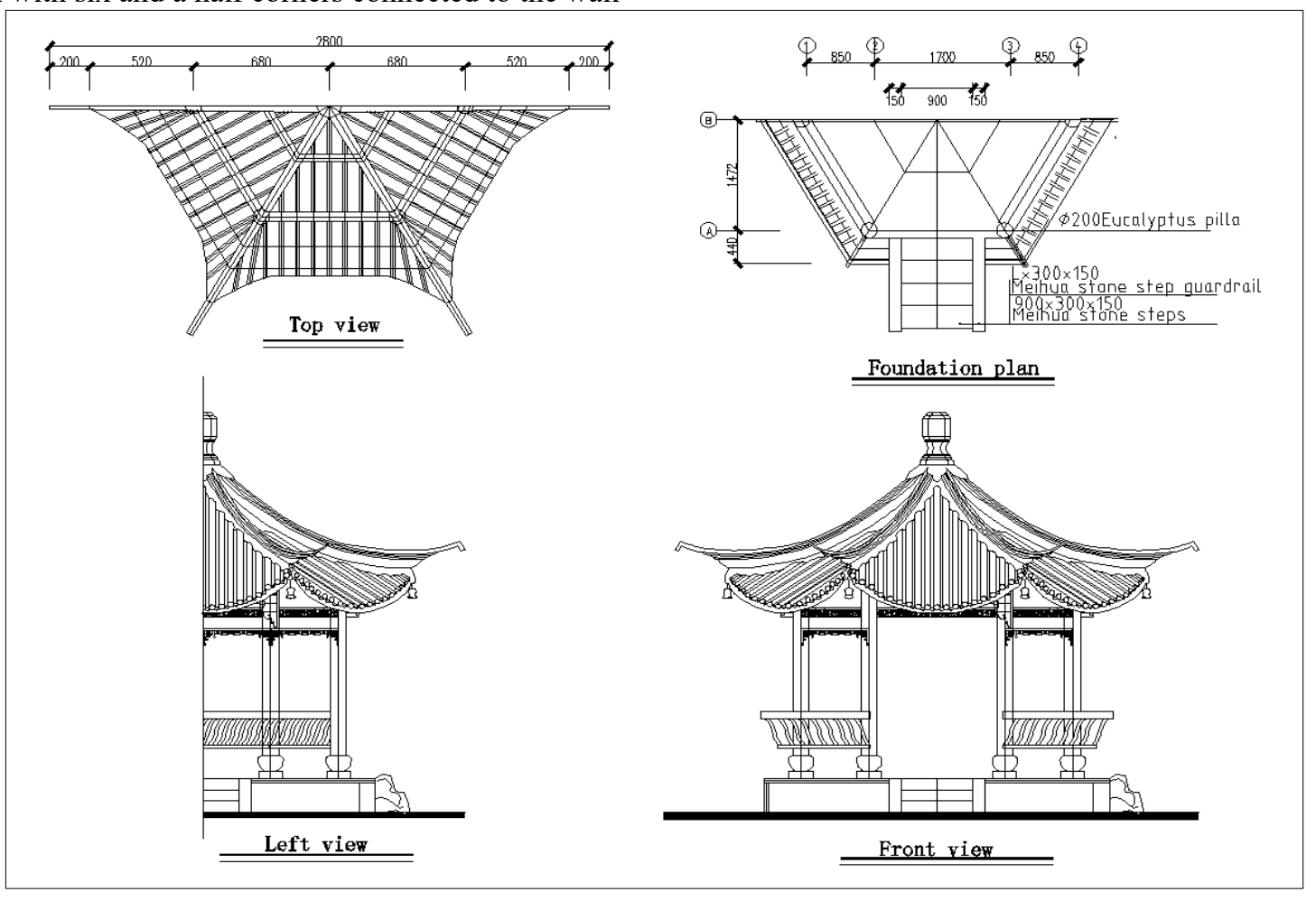

Figure 7. a half pavilion at hexagon 
5.4.2. Drilling wood to make fire. At the entrance of the exhibition area, a temperature sensing switch is installed. When the audience passes through the ancient hole (as shown in Figure 8), the original ecological environment in the hole immediately comes alive. Primitive people also began to "drill wood for fire" performance, and with the performance, the fire gradually "lit up". This scene under the control of inductive intelligence can give people a sense of immersive experience and deepen the experience function of the museum. The shape of the cave is shown

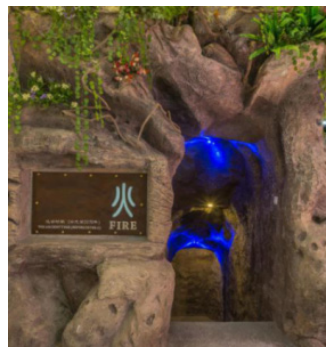

Figure 8. ancient portal

in Figure 9 drilling wood to make fire in the cave. The construction dimension of the opening is shown in Figure 10. It can be seen from the front elevation of the portal modeling that the height and width of the entrance are 2 meters and 0.7 meters, and only one person is allowed to enter. After passing through the hole, it becomes spacious and bright. The ancients on the stone pile are drilling wood for fire. In the aspect of artistic modeling, the concrete is made of cement, brick, steel wire mesh, foam, coating and so on. The temperature sensor is installed at the entrance to control the switch of light and multimedia technology in the hole.

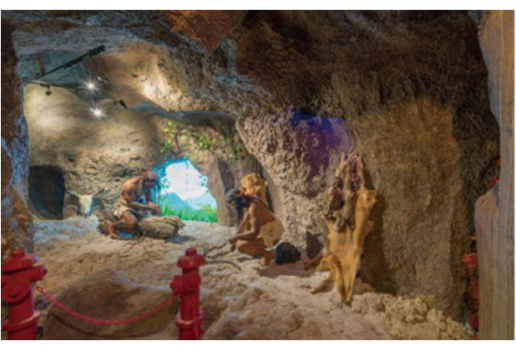

Figure 9. drilling wood to make fire in the cave

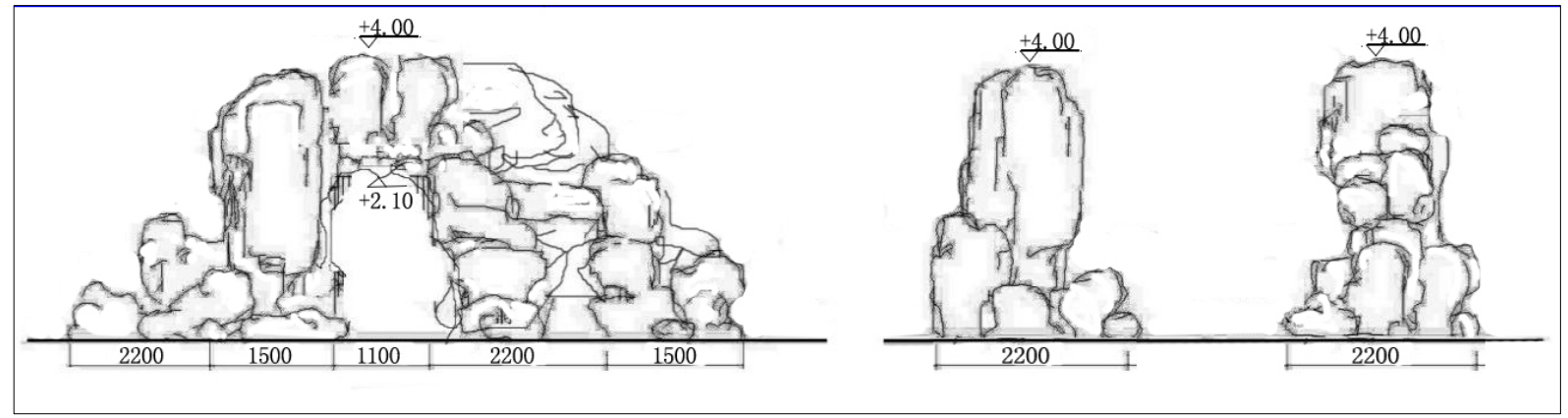

Figure 10. portal construction drawing

5.4.3. Technology and art of fire experience area. There is a fire experience area in the fire exhibition area of ancient residential buildings, so as to achieve the purpose of fire safety, escape skills and other fire education through experience. The experience area adopts simulation technology and integrated control methods of sound, light and electricity to implement intelligent activities such as fire smoke, fire alarm, fire spread, fire rescue, fire extinguisher use, fire induction spray, earthquake escape, first aid training system, etc. Among them, the "Fire Sea" of the "ancient house fire escape experience hall" is composed of electronic atomization, colored lights, multimedia audio and vide. When people touch the fire alarm system, the fire and combustion sound in the simulated fire field are getting louder and louder. At this time, the intelligent escape system is started, and the best escape path is generated according to the location of the fire point, which gives real-time indication to the evacuation sign light at the bottom floor, and evacuates people in a dynamic "safe, nearby and balanced guidance" way, so as to achieve rapid and scientific evacuation ${ }^{[7]}$. As shown in Figure 11 is the escape route diagram, and Figure 12 is the fire virtual experience hall, the exhibition hall adopts the artistic means of intelligent control and simulation of fire scenes to realize the fire fighting experience, so that the participants can learn the scientific escape method according to the green indicator light in the fire escape experience. The key to the experience of fire fighting is to feel the danger of fire and the fragility of life, and to cherish the life after "escaping from fire". 


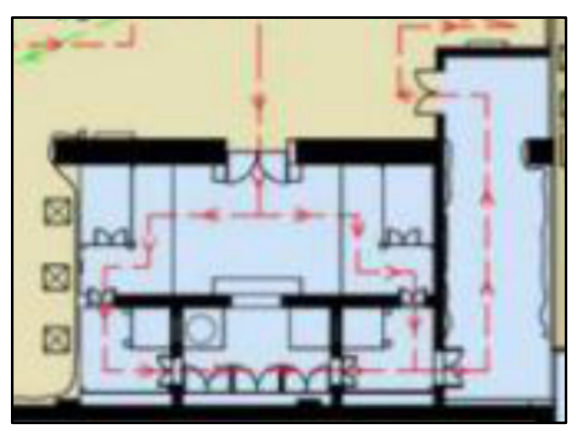

Figure 11. plan of fire experience hall

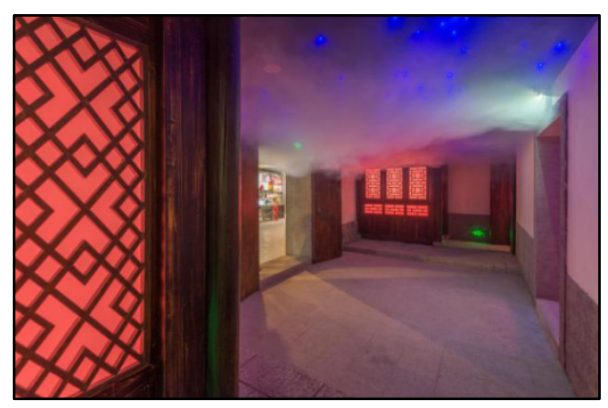

Figure 12. fire experience hall

\section{Epilogue}

Museums have extensive social education functions and indirect economic functions. Having conducted a detailed study and analysis of the exhibition theme of the museum, it combined traditional artistic modeling techniques with scientific and technological modeling, which can break through the difficulties of museum landscape design and make the design innovative. By the above fire museum's half pavilion scene, drilling wood to make fire and fire escape experience project, it concludes that artistic means can help the enhancement of immersive experience, and scientific and technological means can make contributions to the improvement of the depth and dimension of experience. The museum landscape design based on the combination of art and science can facilitate the value elevation of exhibition knowledge, landscape knowledge, exhibition centre education, science, art and interest. The combination of art and science and technology, which is the new trend of landscape design, provides a better comprehensive solution for the innovation in museum landscape design.

\section{References}

1. Wang Mu.(2006,12) Functional interpretation of landscape design. Decoration,32(6):215-218.

2. Wu Zhong.(2017,07) Landscape design [D] Wuhan University Press, Wuhan.

3. Website of the supervision department of the Central Commission for Discipline Inspection $(2015,06,01)$ [enter the museum to inherit Chinese civilization] Nantong Museum, the first public museum in China. http://www.ccdi.gov.cn/lswh/wenhua/wenyuan/20 1511/t20151117_156455.html

4. Liu Changqing, $\mathrm{Pu} \mathrm{Xu.} \mathrm{(2017,09} \mathrm{)Problems} \mathrm{and}$ countermeasures of modern landscape design [J] popular literature and art:117-118.

5. Gao Zhuoyu. Xiang Yanqiong. $(2019,12)$ Application of Chinese classical garden features and design techniques in Suzhou Museum. Industrial design: 117 .

6. Ministry of housing and urban rural development of the people's Republic of China.(2018)Technical standard for fire emergency lighting and evacuation indication system: GB 50309-2018 . Shenyang Fire Research Institute of Emergency Management Department, Shenyang. 